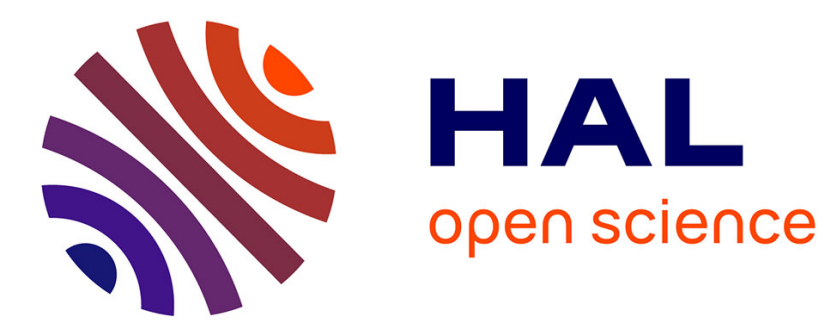

\title{
Soft Matter Drainage in a rising foam
}

Pavel Yazhgur, Emmanuelle Rio, Florence Rouyer, Franck Pigeonneau, Anniina Salonen

\section{To cite this version:}

Pavel Yazhgur, Emmanuelle Rio, Florence Rouyer, Franck Pigeonneau, Anniina Salonen. Soft Matter Drainage in a rising foam. Soft Matter, 2015, 10.1039/C5SM01886B . hal-01235416

\section{HAL Id: hal-01235416 https://hal.science/hal-01235416}

Submitted on 7 Dec 2015

HAL is a multi-disciplinary open access archive for the deposit and dissemination of scientific research documents, whether they are published or not. The documents may come from teaching and research institutions in France or abroad, or from public or private research centers.
L'archive ouverte pluridisciplinaire HAL, est destinée au dépôt et à la diffusion de documents scientifiques de niveau recherche, publiés ou non, émanant des établissements d'enseignement et de recherche français ou étrangers, des laboratoires publics ou privés. 


\section{Soft Matter}

\section{Accepted Manuscript}

This article can be cited before page numbers have been issued, to do this please use: P. Yazhgur, E. Rio,

F. Rouyer, F. Pigeonneau and A. Salonen, Soft Matter, 2015, DOI: 10.1039/C5SM01886B.

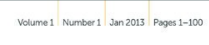

\section{Soft Matter}

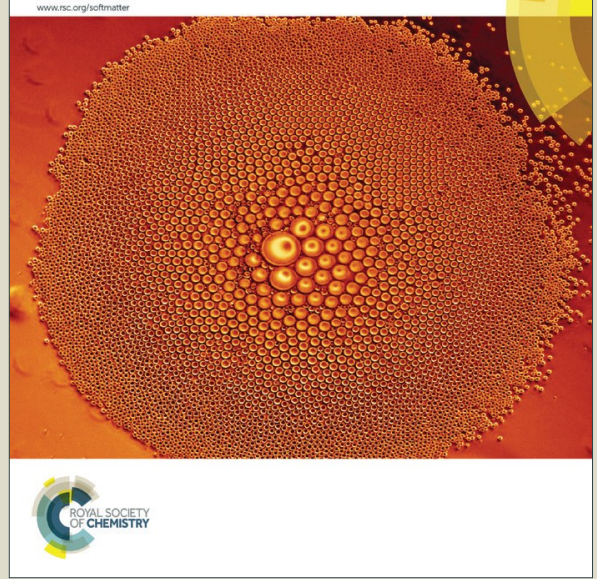

This is an Accepted Manuscript, which has been through the Royal Society of Chemistry peer review process and has been accepted for publication.

Accepted Manuscripts are published online shortly after acceptance, before technical editing, formatting and proof reading. Using this free service, authors can make their results available to the community, in citable form, before we publish the edited article. We will replace this Accepted Manuscript with the edited and formatted Advance Article as soon as it is available.

You can find more information about Accepted Manuscripts in the Information for Authors.

Please note that technical editing may introduce minor changes to the text and/or graphics, which may alter content. The journal's standard Terms \& Conditions and the Ethical guidelines still apply. In no event shall the Royal Society of Chemistry be held responsible for any errors or omissions in this Accepted Manuscript or any consequences arising from the use of any information it contains. 


\section{Journal Name}

\section{ARTICLE TYPE}

Cite this: DOI: $10.1039 /$ xxxxxxxxxx

Accepted Date

DOI: $10.1039 / x x x x x x x x x x$

www.rsc.org/journalname

\section{Drainage in a rising foam}

\author{
Pavel Yazhgur, ${ }^{a}$ Emmanuelle Rio, ${ }^{a}$ Florence Rouyer, ${ }^{b}$ Franck Pigeonneau ${ }^{c}$ and Anniina \\ Salonen ${ }^{a}$
}

\begin{abstract}
Rising foams created by continuously blowing gas into a surfactant solution are widely used in many technical processes, such as flotation. The prediction of the liquid fraction profile in such flowing foams is of particular importance since this parameter controls the stability and the rheology of the final product. Using drift flux analysis and recently developed semi-empirical expressions for foam permeability and osmotic pressure, we build a model predicting the liquid fraction profile as a function of height. The theoretical profiles are very different if the interfaces are considered as mobile or rigid, but all of our experimental profiles are described by the model with mobile interfaces. Even the systems with dodecanol, which are well known to behave as rigid in forced drainage experiments. This is because in rising foams the liquid fraction profile is fixed by the flux at the bottom of the foam. Here the foam is wet with higher permeability and the interfaces are not in equilibrium. These results demonstrate once again that it is not only the surfactant system that controls the mobility of the interface, but also the hydrodynamic problem under consideration. For example liquid flow through the foam during generation or in forced drainage is intrinsically different.
\end{abstract}

\section{Introduction}

Foams are ubiquitous in many technical processes, such as flotation or oil recovery ${ }^{1,31}$. They are also present in varied forms in our daily lives, as soft solids or fluids, in food and beverage products as well as in insulating materials. The physical properties of foams are largely controlled by the bubble size $R$ and the size distribution and the liquid fraction, ratio of the liquid volume to the foam volume: $\varepsilon=V_{l i q} / V_{\text {foam }}$, which makes them such important parameters. For example, the yield stress of a foam which ensures that the foam can sustain its own weight, is proportional to $\left(\varepsilon_{\max }-\varepsilon\right)^{2} / R^{1}$, with $\varepsilon_{\max }=0.36$, so that small bubbles are necessary to obtain a self-standing foam. This is why depending on the application, $R$ and $\varepsilon$ should take rather specific values (see Figure 1(a) for examples).

Despite their importance, the control of these two parameters during the foam generation remains mainly empirical ${ }^{5}$. In general, with a given foam generation process, the bubble size and the liquid fraction can only be varied in a rather narrow range. Moreover there is often a correlation between the two parameters, and generation methods where they can be varied indepen-

\footnotetext{
${ }^{a}$ Laboratoire de Physique des Solides, CNRS and Université Paris-Sud, 91405 Orsay Cedex, France. E-mail: pavel.yazhgur@u-psud.fr

${ }^{b}$ Laboratoire Navier, Université Paris-Est, 5 Bd Descart, 77454 Champs-sur-Marne, France.

c Surface du Verre et Interfaces, UMR 125 CNRS/Saint-Gobain, 39 quai L. Lefranc, Aubervilliers, France.
}

dently are scarce. Examples are shown in figure 1(b), where the bubble size and the liquid fraction are shown for foams prepared by blowing gas thorough a porous media (rising foam) and by the turbulent mixing of liquid and gas jets (turbulent mixing, the method is described in ${ }^{24}$ ). A strong correlation between bubble size and liquid fraction is observed, which has also been shown in foams generated by microfluidic techniques ${ }^{8}$. Finally, the different methods allow for the exploration of a range of bubble radii and liquid fractions, but rarely independently. In general either the liquid fraction or the bubble size is controlled and sets the other in combination with the flow conditions. It is only through a fine understanding of each of the processes used that control over the bubble radius and the liquid fraction, and thus, of foam properties can be achieved.

In the following, we focus on a very common technique used to generate foams: blowing gas through a porous media. The process can be divided into two separate steps. The first one is the formation and rising of the bubbles in the liquid before they agglomerate at the liquid/air interface. The second is the simultaneous rising and drying of the foam. The bubble radius is mainly controlled during the first stage. Theoretical descriptions of this process can be found in the literature for a number of different techniques, however some aspects still remain unclear ${ }^{5,15}$. In this article we choose to measure the size of the bubbles and to focus on the second stage, in which the liquid fraction profile in the foam is fixed. This is set by a balance between the foam rising in the column due to the buoyancy force and the gravity driven 
drainage of the liquid leading to foam drying. After a certain time, a steady-state regime can be reached, characterized by a stationary liquid fraction profile.

Although a thorough understanding of the second step is essential for a description of the final foam, there are only few theoretical publications ${ }^{11,12,21,27,28}$ in this field and experimental data concerning this problem are scarce ${ }^{6,29}$. Among these works is the model developed by Stevenson to establish the liquid fraction profile as a function of the gas flow rate imposed at the bottom $^{27,28}$. The basis of this model is that the liquid fraction profile is mainly fixed by the drainage of the foam during its generation. The drainage is quantified by the permeability $k$ of the porous media formed by the foam, which sets the drainage velocity for a given pressure drop through the Darcy's law (Equation 6). The permeability is then expressed as $k=m \varepsilon^{n}, m$ and $n$ being empirical parameters.

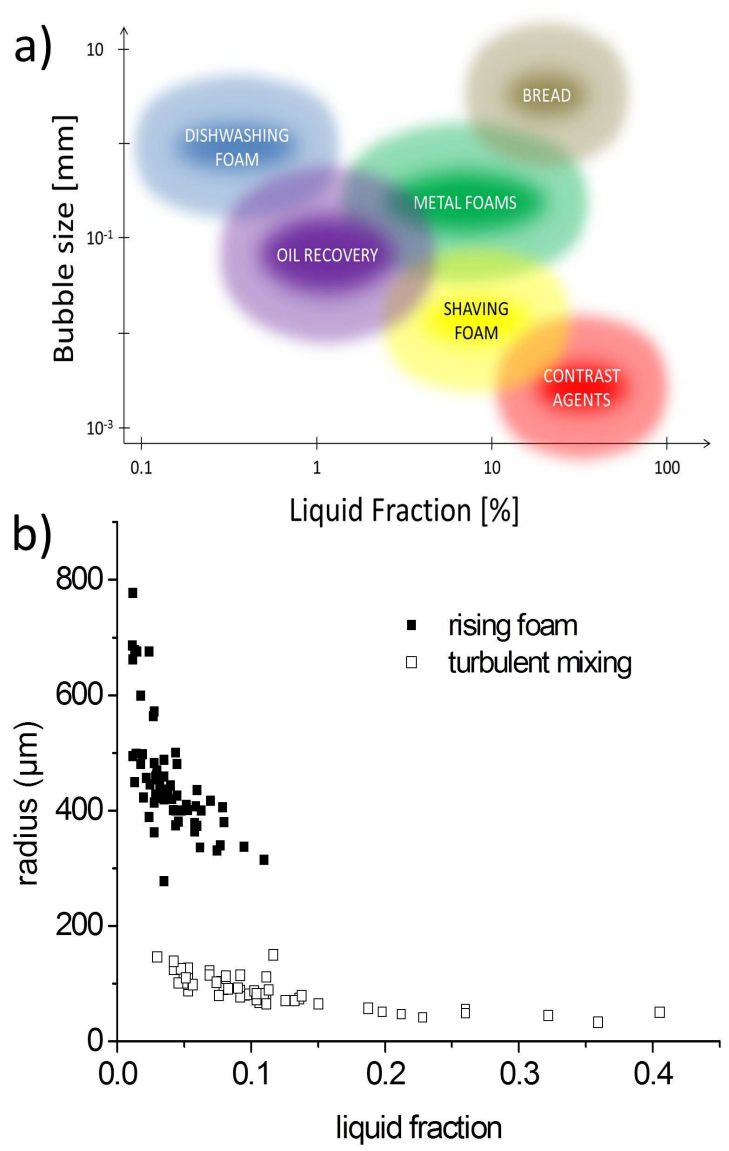

Fig. 1 (a) Different domains of applications of foams in industrial processes together with the typical bubble sizes and liquid fractions used. (b) The relation between the radius and the liquid fraction for two different generation methods.

It is well-known that drainage in foams is quite different depending on their physical-chemistry. The dissipation at the interfaces is indeed non negligible and can be taken into account by writing the tangential stress at the liquid/gas interface ${ }^{2}$. This surface stress is the sum of a viscous component and of an elastic component equal to the surface tension gradient at the liquid/air interface. In foam drainage, it is generally accepted ${ }^{3,26}$ that the viscous term is dominant because surface tension is expected to be homogeneous. The importance of the surface viscosity $\mu_{s}$ is then quantified by the ratio between the dissipation in the bulk and at the interface, i.e. the Boussinesq number $B o=\frac{\mu_{s}}{\mu R_{P b}}$ with $\mu$ the bulk viscosity and $R_{P b}$ the radius of the Plateau borders 3,16 . Large $B o(B o>>1)$ leads to a large dissipation at the interfaces, hence rigid interfaces, whereas small $B o(B o<<1)$ leads to dissipation mainly in the bulk, hence mobile interfaces.

In this article, our goal is to explore the impact of physicalchemistry on foam generation. In a theoretical section, we recall Stenvenson's model and build on it to explore more quantitatively the impact of physical-chemistry on the surface rigidity. We implement a semi-empirical law for the permeability and the foam structure. For the permeability $k$, we use $k=m(\varepsilon) \varepsilon^{n}$ where the power $n$ is equal to 2 for rigid interfaces and to $3 / 2$ for fluid ones ${ }^{3,26}$. The function $m(\varepsilon)$ has been obtained over a large range of $\varepsilon$ from drainage measurements in forced drainage and fluidized bed experiments both in the fluid or in the rigid limit ${ }^{23}$. For the foam structure, we use recently developed semi-empirical expressions for the osmotic pressure ${ }^{9,19}$. This allows us to predict the liquid fraction profile of the foam as a function of the bubble radius and the gas flow rate both in the rigid and in the mobile limits.

We present experiments done with solutions of surfactants, that are known to lead to rigid or fluid interfaces in drainage experiments ${ }^{23,26}$. The bubble radius and the liquid fraction profile of the foam are measured for each foaming solution. Our work provides a systematic comparison between the liquid fraction profile obtained in the model and in the experiments. Moreover, we show that every surfactant solution used in this work leads to a liquid fraction profile well described by the equations obtained in the mobile limit. We show that this is because the forced drainage experiments are performed on dry foams with old interfaces whereas in the foamability experiments one cannot get around wet foams and newly created interfaces.

\section{Materials and methods}

A commercially available device Foamscan (Teclis, France) is used to generate and characterize the foams (see Figure 2(a)). This machine produces foam by blowing gas through a porous frit at a controlled gas flow rate varying from 5 to $400 \mathrm{ml} / \mathrm{min}$. To avoid coarsening of bubbles nitrogen with traces of water-insoluble $C_{6} F_{14}$ is used in the experiments. The cell used has a square cross section $\left(2.5 \times 2.5 \mathrm{~cm}^{2}\right)$ and a height of $30 \mathrm{~cm}$.

\subsection{Liquid fraction measurement}

The liquid fraction is calculated from electrical conductivity measurements. Pairs of electrodes measure a reference conductivity of the liquid at the bottom of the cell $\sigma_{l}$ and a foam conductivity $\sigma_{f}$ at five different positions (see Figure 2(a)). To avoid electrolysis an alternating current is used with a frequency of $1 \mathrm{kHz}$ and a voltage of $1 \mathrm{~V}$. At the chosen frequency the capacitance of the foam can be neglected and the active resistance can be directly measured $^{1}$. 


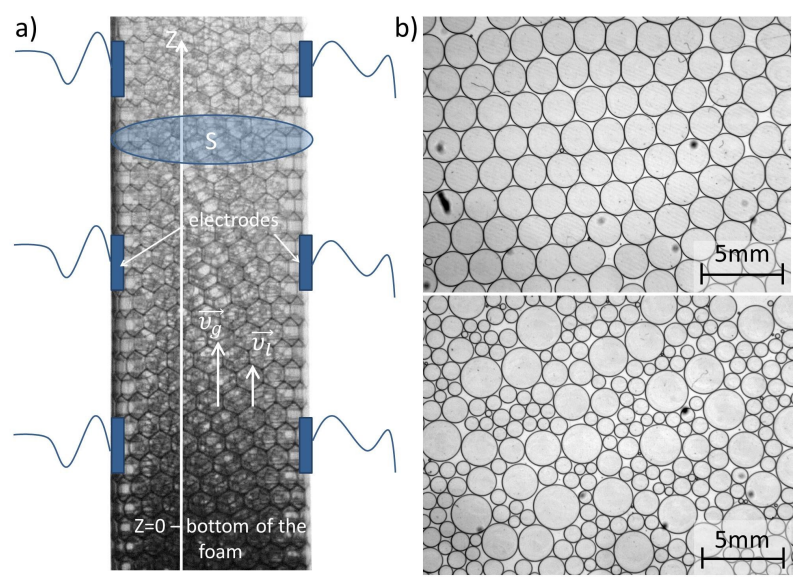

Fig. 2 On the left: a schema of the experimental set-up, the $z$ axis is defined along the direction of the tube. On the right: bubbles squeezed between two glass plates sampled from monodisperse (at the top) and bidisperse foam (at the bottom)

For foams the relative conductivity $\sigma=\sigma_{f} / \sigma_{l}$ is found to be primarily a function of the liquid fraction $\varepsilon$ defined as the ratio between the volume of liquid and the total volume of the foam. A semi-empirical relation can then be used to calculate $\varepsilon$ from experimentally measured $\sigma^{7}$ :

$$
\varepsilon=\frac{3 \sigma(1+11 \sigma)}{1+25 \sigma+10 \sigma^{2}} .
$$

It has been shown that this empirical relation describes experimental data very well over the whole range of $\varepsilon$ and $\sigma$ (both ranging from 0 to 1 ).

To keep the liquid level constant the set-up is in contact with a big vessel containing the same foaming solution. By varying the level of liquid the position of the interface between the foam and the solution can be moved relative to the electrodes. In this way different heights in the foam can be probed and a full liquid fraction profile can be measured.

A steady-state defined by a constant conductivity is reached before each measurement of the liquid fraction.

\subsection{Bubble size measurement}

To measure the bubble size distribution a sample of foam is collected from the column and squeezed between two glass plates separated by $h=150 \mu \mathrm{m}$ (Figure 2(b)). The cross-sectional area $A$ of the squeezed bubble is measured from photos. Assuming volume conservation, the radius $R$ of each bubble can be easily calculated $^{18}$ :

$$
R=\sqrt[3]{\frac{3 A h}{4 \pi}},
$$

The bubbles are produced by a polydisperse porous frit so the obtained size distribution strongly depends on the gas flow rate (Figure 2(b)). We use the Sauter mean radius $R_{32}=\left\langle R^{3}\right\rangle /\left\langle R^{2}\right\rangle$ as the characteristic bubble radius.

\subsection{Surface tension measurement}

The surface tension of the foaming solutions in time is measured by bubble profile analysis tensiometry using a commercially available device (Tracker, Teclis, France).

\subsection{Foaming solutions}

Two different surfactants have been used, sodium dodecyl sulphate (SDS) at $12 \mathrm{~g} / \mathrm{L}$ (approximately 5 times the critical micelle concentration) and tetradecyltrimethylammonium bromide (TTAB) at $3 \mathrm{~g} / \mathrm{L}$ (approximately 2 times the critical micelle concentration). The different foaming solutions used are listed below, with the name by which they are referred to in the rest of the text:

- SDS at $12 \mathrm{~g} / \mathrm{L}$ - SDS12

- SDS at $12 \mathrm{~g} / \mathrm{L}$ with $0.3 \mathrm{~g} / 1$ of dodecanol - SDS12-DOH3

- SDS at $12 \mathrm{~g} / \mathrm{L}$ with $0.04 \mathrm{~g} / 1$ of dodecanol and $0.1 \mathrm{M}$ of $\mathrm{NaCl}$ - SDS12-DOH4-NaCl

- $\mathrm{TTAB}$ at $3 \mathrm{~g} / \mathrm{L}$ with $0.2 \mathrm{~g} / \mathrm{l}$ of dodecanol - TTAB3-DOH2

All chemicals were purchased from Sigma-Aldrich and used as received. All solutions are prepared with deionized Milli-Q water $(18.2 \mathrm{M} \Omega \cdot \mathrm{cm})$. The samples are used within one week of preparation to avoid SDS hydrolysis.

All experiments are performed at room temperature $20 \pm 2^{\circ} \mathrm{C}$.

\section{Theoretical background}

Let us consider a foam which moves inside a tube with a crosssectional area $S$ (see figure 2). The $z$ axis is oriented along the column in the direction of the foam flow. The bottom of the foam corresponds to $z=0$. At each point the foam can be fully characterized by the liquid fraction $\varepsilon(z, t)$, the mean radius of the bubbles $R(z, t)$, and the flow rates of liquid $\mathrm{Q}_{l}(z, t)$ and gas $\mathrm{Q}_{g}(z, t)$. In this case the linear velocities of liquid $v_{l}(z, t)$ and gas $v_{g}(z, t)$ can be calculated:

$$
\begin{gathered}
\overrightarrow{v_{l}}=\frac{\overrightarrow{Q_{l}}}{S \varepsilon}, \\
\overrightarrow{v_{g}}=\frac{\overrightarrow{Q_{g}}}{S(1-\varepsilon)} .
\end{gathered}
$$

The liquid and gas do not travel at the same speed and this difference is used to define the slip velocity:

$$
\overrightarrow{v_{s}}=\overrightarrow{v_{l}}-\overrightarrow{v_{g}}
$$

This slip velocity can also be named drainage velocity as it corresponds to the liquid flow through the bubble assembly. This problem has been extensively investigated by free and forced drainage experiments. The general approach in these studies is to link the system under investigation to a liquid flowing through a porous media ${ }^{1}$. An expression for the flow rate of the fluid through porous media can be calculated by solving the NavierStokes equations. In the case of laminar flow, where inertia is negligible compared to the viscous drag, the flow equation is well known as Darcy's law: 


$$
\frac{\overrightarrow{Q_{s}}}{S}=\overrightarrow{v_{s}} \varepsilon=-K \vec{\nabla} P_{d} / \mu
$$

where $\mu$ is the viscosity of the liquid, $\vec{\nabla} P_{d}$ is the dynamic pressure gradient, $R$ is the radius of bubbles and $K$ the permeability coefficient. It is generally accepted that $K$ is proportional to the square of the bubble radius and can be expressed as:

$$
K=R^{2} k
$$

where $k$ depends only on the liquid fraction and on the surface mobility.

Combining Equations 5 and 6, we get :

$$
\overrightarrow{Q_{l}}=\frac{\varepsilon \overrightarrow{Q_{g}}}{(1-\varepsilon)}-\frac{S R^{2} k(\varepsilon)}{\mu} \vec{\nabla} P_{d}
$$

Now let us assume that our foam has reached a stationary regime in which the liquid fraction does not depend on the time, i.e. $\partial \varepsilon / \partial t=0$. We know from the experiments that such a regime exists. To avoid misunderstanding let us mention that $\varepsilon$ is still a function of $z$. In the steady state, assuming that there is no evaporation in the system, we easily get from mass conservation that the flow rates of gas and liquid are constant along the tube $\partial Q_{g} / \partial z=\partial Q_{l} / \partial z=0$. It means that in the stationary regime the flow rates of gas and liquid at any point inside the foam are equal to the ones used to produce the bubbles. In the case of rising foam we can control $Q_{g}$, while $Q_{l}$ is self-adjusted to satisfy Equation 8 .

In our particular case of rising foam the dynamic pressure gradient includes gravity and capillary forces:

$$
\vec{\nabla} P_{d}=-\rho \vec{g}-\vec{\nabla} \Pi,
$$

where $\rho$ is the density of the liquid phase and $\Pi$ is the osmotic pressure in the foam ${ }^{1}$.

Substituting Equation 9 in Equation 8 we get:

$$
\overrightarrow{Q_{l}}=\frac{\varepsilon \overrightarrow{Q_{g}}}{(1-\varepsilon)}+\frac{S R^{2} k(\varepsilon)}{\mu}(\rho \vec{g}+\vec{\nabla} \Pi) .
$$

Applying the mass conservation condition $\partial Q_{l} / \partial z=0$ to Equation 10 we can eliminate $Q_{l}$ and get a differential equation which can be solved to get the liquid fraction profile $\varepsilon(z)$. But the corresponding equation is of second order and is quite complicated. An elegant way to simplify the calculations has been proposed by Stevenson ${ }^{30}$. Far away from the bottom the osmotic pressure gradient can be neglected and the liquid fraction $\varepsilon^{\infty}$ is almost constant with height. Then $Q_{l}$ is a function only of $\varepsilon^{\infty}$ and the stationary state corresponds to the condition $\partial Q_{l} / \partial \varepsilon^{\infty}=0^{30}$. Assuming a constant bubble radius, this condition can be written as:

$$
\frac{\mu Q_{g} / S}{\left(1-\varepsilon^{\infty}\right)^{2} R^{2} \rho g}=\frac{\partial k\left(\varepsilon^{\infty}\right)}{\partial \varepsilon^{\infty}} .
$$

This equation can be solved numerically to get $\varepsilon^{\infty}$ and calculate $Q_{l}$ from Equation 10 taking $\vec{\nabla} \Pi=0$. The obtained value of $Q_{l}$ can be then used to calculate the whole liquid fraction profile from Equation 10 with $\vec{\nabla} \Pi \neq 0$. This differential equation is of first order and can be easily solved numerically.

To perform the calculations appropriate expressions for permeability and pressure gradient are required. In the following, we benefit from recent progress in understanding foam structure and foam permeability to propose semi-empirical expressions for both quantities.

There are two generally accepted models for predicting foam permeability. In the rigid limit, the dissipation takes place mainly in the Plateau borders ${ }^{32}$. In the fluid limit, it is presumed that there are no viscous losses in the Plateau borders and that dissipation occurs only in the nodes instead. In the first limit the theory gives $k \propto \varepsilon^{2}$ whereas the second limit leads to $k \propto \varepsilon^{3 / 213}$. The drainage equation can be solved in these two limits, even analytically in the first case.

To improve agreement between theory and experiments some mixed models which take into account permeabilities both in Plateau borders and in nodes have been proposed ${ }^{14,22}$. Unfortunately, no analytical expression for $k$ is available. But, recent semi-empirical expressions describing experimental data very well in a wide range of liquid fractions were proposed ${ }^{23}$ for both limits of mobile and rigid interfaces:

$$
\begin{aligned}
& k(\varepsilon)=\frac{\varepsilon^{3 / 2}}{425\left(1-2.7 \varepsilon+2.2 \varepsilon^{2}\right)^{2}} \text { (mobile interface), } \\
& k(\varepsilon)=\frac{\varepsilon^{2}}{312\left(1-2.15 \varepsilon+1.37 \varepsilon^{2}\right)^{2}} \text { (rigid interface), }
\end{aligned}
$$

which we will use in the following.

Once again, we will use a semi-empirical relationship to express the osmotic pressure $\Pi$ :

$$
\Pi=\alpha \frac{\left(\varepsilon_{\max }-\varepsilon\right)^{2}}{\sqrt{\varepsilon}} \frac{\gamma}{R}
$$

with $\alpha=7.3, \varepsilon_{\max }=0.26$ for ordered foams ${ }^{9}$ and $\alpha=3.2, \varepsilon_{\max }=$ 0.36 for disordered ones ${ }^{19}$. Polydispersity in the bubble size can stop the bubbles from crystallising leading to a disordered packing. Therefore we refer to $\varepsilon_{\max }=0.26$ as monodisperse and $\varepsilon_{\max }=0.36$ as polydisperse.

Using the above mentioned expressions for permeability and osmotic pressure Equation 10 can be solved numerically in the two limiting cases of mobile and rigid interfaces to get a liquid fraction profile $\varepsilon(z)$. To perform the integration we need an appropriate boundary condition. We assume that at the bottom of the foam bubbles are not deformed and the boundary condition can be written as $\varepsilon(z=0)=\varepsilon_{\max }$.

\section{Results and discussion}

To check the applicability of the developed theory, the liquid fraction was measured for different foaming solutions at different gas flow rates.

Experiments with a solution of SDS at $12 \mathrm{~g} / \mathrm{l}$ are shown in Figure 3. The bubble size distribution, shown in the inset, is monomodal and sharply peaked. Using the mean Sauter radius $\left(R_{32}=523 \mu \mathrm{m}\right)$ liquid fraction profiles are calculated in the rigid 
(Equation 13) and in the mobile (Equation 12) limits. For each limit, profiles for monodisperse and polydisperse foams are calculated using the corresponding values for $\alpha$ and $\varepsilon_{\max }$ in the Equation 14. This results in 4 different theoretical curves. We can see that rigid and mobile limits predict significantly different liquid fractions especially far away from the bottom of the foam (Figure 3). Thus, it should not be a problem to discriminate between the mobile or the rigid limit from the experimental data. However for a given mobility the curves corresponding to ordered and polydisperse limits almost coincide. The ordering at the bottom of the foam thus results in such fine variations that they are not detected by our experiments. That is why in the following we will consider only the case of monodisperse ordered foams. Experimental data shown with black points agrees well with the model of mobile interfaces. An estimation of error bars is discussed in the Appendix A. The obtained result is expected. It is well known, both from interfacial rheology and from drainage experiments that newly prepared SDS solutions have low surface shear viscosity (between $10^{-8}$ and $10^{-7} \mathrm{~kg} / \mathrm{s}^{4}$ ) and produce mobile interfaces in both forced and free drainage experiments ${ }^{25}$.

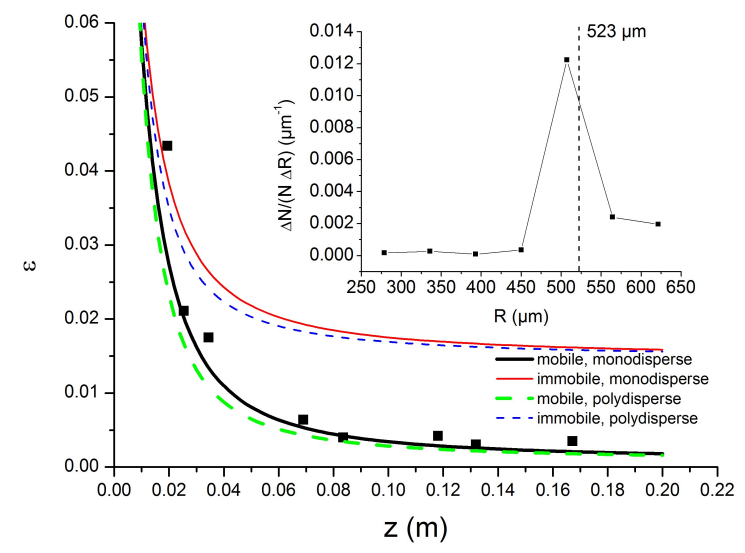

Fig. 3 Liquid fraction profile for SDS12 foam produced with a $10 \mathrm{ml} / \mathrm{min}$ gas flow rate. Black points represent experimental data, the lines show calculated profiles in different limits using the semi-empirical relations for permeability in equations 12 and 13, but with no adjustable parameters. Inset: Number weighted bubble size distribution.

Increasing the flow rate to $30 \mathrm{ml} / \mathrm{min}$ we change the bubble size distribution drastically as smaller holes in the frit become activated. The bubble size distribution weighted by number becomes bimodal as shown in the inset of Figure 4. In this case, it is not evident which mean value is the best to input in our calculations as a bubble size. Thus, we have chosen three different ways to determine the mean size: a mode size corresponding to small bubbles ( $303 \mu \mathrm{m}$ ), a mean Sauter radius $R_{32}(457 \mu \mathrm{m})$ and a mode corresponding to big bubbles $(558 \mu \mathrm{m})$. For each mean value liquid fraction profiles are calculated (see Figure 4). Only curves corresponding to the mobile limit are shown, as the rigid model predicts much higher liquid fractions than the ones measured experimentally. The best agreement with the experiments is obtained using the big bubble radius as input. It seems that the big bubbles control the foam permeability even if their number is very small. This is both because their total volume is actually non negligible and because the liquid tends to follow the path of lower permeability.

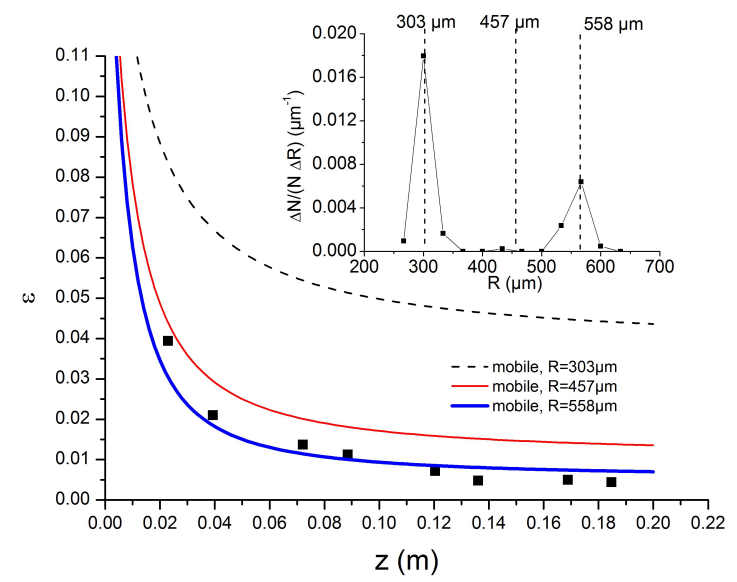

Fig. 4 Liquid fraction profile for the SDS12 foam produced with a 30 $\mathrm{ml} / \mathrm{min}$ gas flow rate. Black points represent experimental data, the lines show calculated profiles for different bubble sizes. Inset: Number weighted bubble size distribution.

Summing up we can conclude that the model with mobile interfaces captures the liquid fraction profile for foams stabilized by fresh solutions of SDS. We have also shown that the liquid fraction profile is not very sensitive to the polydispersity of bubbles making the model valid for both monodisperse and polydisperse foams. Moreover in the case of bidisperse foam with two distinct populations of bubble sizes, the largest bubble size has to be used in the model for it to agree with the experimental liquid fraction profile. Because our foam typically has very narrow bubble size distributions, we will focus only on the monodisperse case in the following discussion.

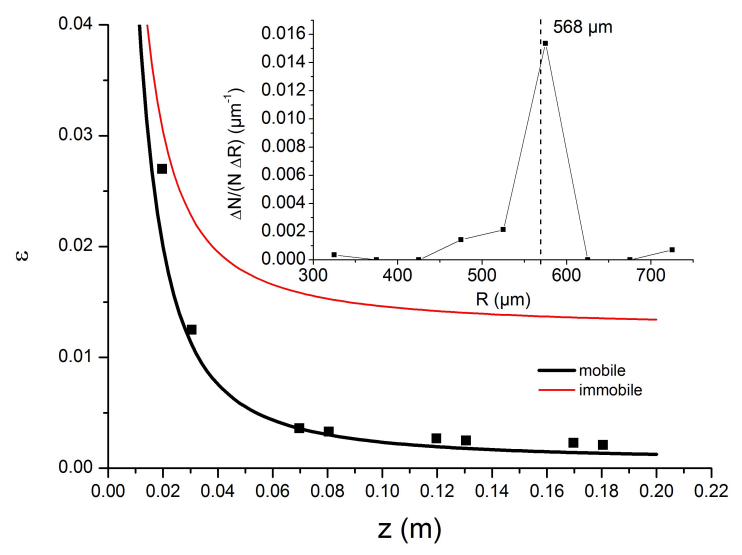

Fig. 5 Liquid fraction profile for the SDS-DOH3 foam produced at 10 $\mathrm{ml} / \mathrm{min}$ gas flow rate. Black points represent experimental data, the lines show calculated profiles in different limits. Inset: bubble size distribution. 
The liquid fraction profile was expected to be rather different with higher surface viscosities. The influence of surface rheology on the foamability has already been observed in literature, with fatty acids/surfactant mixtures, for example ${ }^{20}$. To increase the surface viscosity, mixtures of SDS and dodecanol are commonly used. It is well known from forced drainage experiments that the addition of dodecanol increases the surface viscosity (up to approximately $10^{-6} \mathrm{~kg} / \mathrm{s}$ ) and makes the interfaces more rigid ${ }^{24}$. One could expect data with such a mixture to agree with the rigid model. As we can see in Figure 5 this is not the case. The system is well described by the model supposing mobile interfaces, exactly as with pure SDS. Other systems shown to have rigid interfaces in forced drainage experiments ${ }^{18}$ were tested, but they are all well described by the mobile limit (see Figure 6). The foams from TTAB3-DOH2 with the smallest bubble size are slightly wetter than predicted by the model, suggesting that for this system the interfaces are marginally rigid. But they are still far from being described by the rigid model.

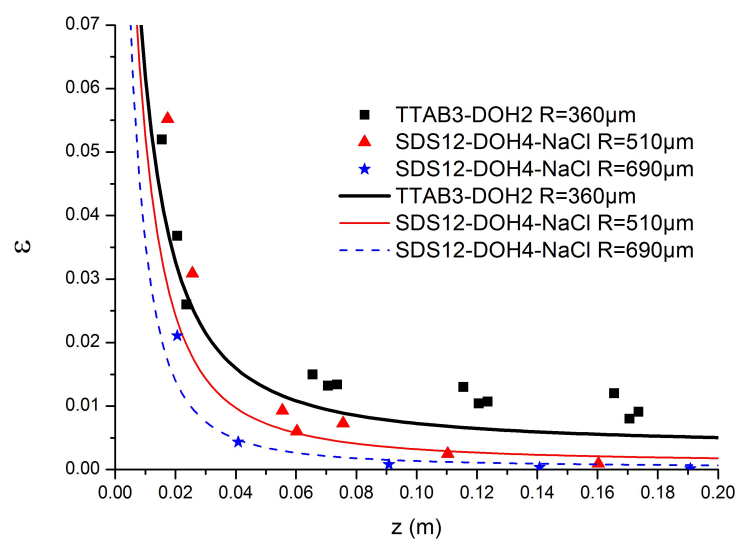

Fig. 6 Liquid fraction profile for different "rigid" foams produced at 10 $\mathrm{ml} / \mathrm{min}$ gas flow rate. The lines show calculated profiles in the mobile limit.

To understand this striking result, let us consider the surface stresses on a section of vertical Plateau border surface inside the foam. The continuity of the stress tensor gives us the following boundary condition at the liquid/air interface:

$$
\mu \frac{\partial v}{\partial x}=\frac{\partial \gamma}{\partial z}+\mu_{s} \frac{\partial^{2} u_{s}}{\partial y^{2}}
$$

The term on the left hand side corresponds to the bulk viscous stress. The first term on the right hand side corresponds to the Marangoni stress, the second one shows the influence of the interfacial viscous stress.

In a forced drainage experiment it is generally assumed that the surface tension is constant everywhere and that only the surface viscous stress plays a role. In this limit the Marangoni stress is neglected in Equation 15. The bulk and the surface viscous stresses are then compared through the dimensionless Boussinesq number $B o$. Note that $B o$ is expected to vary with the position in the foams because $R_{P b}$ depends on both the liquid fraction and the radius of the bubbles. To calculate $B o$, we use the Plateau border curvature $R_{P b}$ as a characteristic length scale. To estimate $R_{P b}$, we use a relationship between the radius of the bubbles, the radius of Plateau border curvature and the liquid fraction which has been computed for Kelvin foam structure with Surface Evolver ${ }^{22}$ :

$$
\varepsilon=0.332\left(\frac{R_{P b}}{R}(1-\varepsilon)^{1 / 3}\right)^{2}+0.540\left(\frac{R_{P b}}{R}(1-\varepsilon)^{1 / 3}\right)^{3} .
$$

This equation can be solved numerically to get $R_{P b}(\varepsilon)$ for a given bubble size. This dependence can be converted to $R_{P b}(z)$ using the best fit of the liquid fraction profile. The values for $B o$ as a function of foam height are plotted in Figure 7 with two bounding values of $\mu_{s}, 6 \times 10^{-8} \mathrm{~kg} / \mathrm{s}$ for bare SDS and $2 \times 10^{-6} \mathrm{~kg} / \mathrm{s}$ for SDS with $0.3 \mathrm{~g} / \mathrm{L} \mathrm{DOH} \mathrm{from}{ }^{4}$. Already at the bottom of the foam we find $B o \sim 0.2$ and $\sim 5$ for the foams stabilised with SDS and SDS-DOH respectively. This leads to the classical conclusion which expects different mobilities for the dodecanol laden and free interfaces, but this is not what we observe (Figure 5).

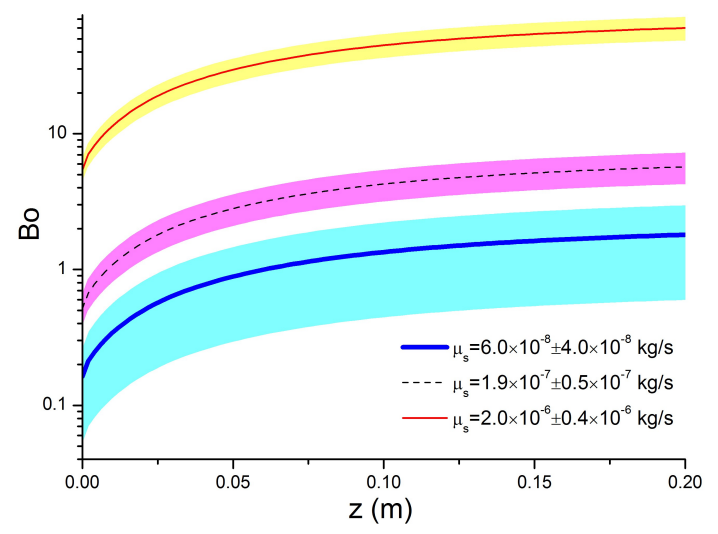

Fig. 7 Boussinesq $B$ o numbers for a "mobile" $\left(\mu_{s}=6 \times 10^{-8} \mathrm{~kg} / \mathrm{s}\right.$ for SDS solution) and two "rigid" ( $\mu_{s}=2 \times 10^{-6} \mathrm{~kg} / \mathrm{s}$ for SDS with $0.3 \mathrm{~g} / \mathrm{l}$ DOH and $1.9 \times 10^{-7} \mathrm{~kg} / \mathrm{s}$ for SDS with $0.1 \mathrm{~g} / \mathrm{l} \mathrm{DOH}$ ) surface viscosities vs height. The surface viscosity data are taken from ${ }^{4}$.

A critical difference between our experiments and drainage experiments is that we continuously generate new interfaces, as new bubbles are formed at the bottom. Knowing that the dodecanol, which is expected to rigidify the interfaces, takes time to adsorb means that the interfaces at the bottom of the foam contain less dodecanol than at the top. This has two consequences: there will be a difference in surface tension and in the surface viscosity between the top and the bottom of the foam. An influcence of adsorption kinetics on foam generation has already been discussed in the literature ${ }^{5,10}$.

To quantify this effect, we performed experiments with a rising bubble and measured the surface tension of pure SDS and SDSDOH3 solutions as a function of time (Figure 8). The adsorption of SDS is so fast that the surface tension of SDS solutions is practically constant from the start of the measurement (around $1 \mathrm{~s}$ ). But, when dodecanol is added to the system (SDS-DOH3) the 
surface tension continues to slightly decrease during hundreds of seconds.

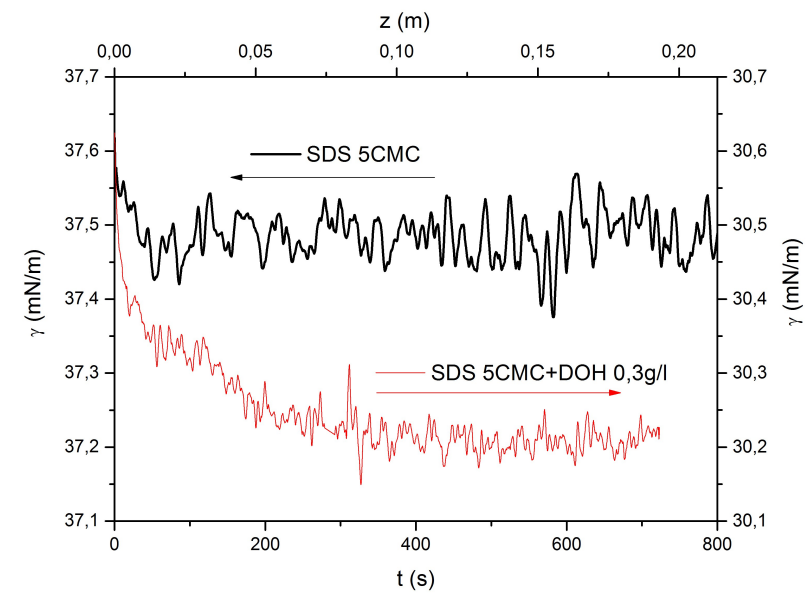

Fig. 8 Surface tension vs time for SDS12 (thick black line) and SDSH12-DOH3 (thin red line). The equivalent height in the foam corresponding to $Q_{g}=10 \mathrm{ml} / \mathrm{min}$ is shown on the top.

Let us come back to the Figure 7 keeping in mind that the adsorption of DOH is slow. If we assume that the adsorption kinetics is similar in the foam and in the pendant drop, we can convert the time dependent surface tension into a function of the altitude in the foam $\left(z=v_{g} t\right)$. The surface tension reaches its equilibrium value at an altitude $z \sim 10 \mathrm{~cm}$ for $Q_{g}=10 \mathrm{ml} / \mathrm{min}$. The change in surface tension at long times is very small (around $0.4 \mathrm{mN} / \mathrm{m}$ ), but the concentration of DOH has a much stronger effect on $\mu_{s}$ than $\gamma$. Indeed at $0.1 \mathrm{~g} / 1 \mathrm{DOH}$ the equilibrium surface tension is equivalent to that with $0.3 \mathrm{~g} / \mathrm{l}$, but $\mu_{s}$ is one order of magnitude smaller $^{4}$ (see Figure 7). Thus, even if a few milliseconds after starting the adsorption experiment (Figure 8), the surface tension almost reached its equilibrium value, it says nothing about the value of the surface viscosity after such a short time. Even a small variation of DOH surface concentration due to adsorption kinetics can drastically change the surface viscosity. Therefore, we think that the slow adsorption is the reason why the interfaces behave as fluid. As shown in Figure 7, a surface shear viscosity as small as $\mu_{s}=1.9 \times 10^{-7} \mathrm{~kg} / \mathrm{s}$ (corresponding to the equilibrium value for SDS with $0.1 \mathrm{~g} / \mathrm{l} \mathrm{DOH}$, i.e. with a slightly smaller surface concentration of dodecanol than in our foaming solution SDSH12-DOH3 at equilibrium) leads to $B o \sim 0.5$ at the bottom of the foam and may explain that the liquid drains as if it has fluid interfaces, which is what we observe.

Before the conclusion, let us note that, in principle, the slow adsorption of DOH should affect both terms on the right hand side of Equation 15 (the influence on the osmotic pressure gradient is negligible see Appendix B). Indeed, a variation of the dodecanol content affects the surface viscosity but it will also lead to surface tension gradients at the interface. This means that a global gradient in surface tension can exist between the top and the bottom of the foam as the interfaces of the bubbles above are older than those below. However, convection due to the drainage flow tends to accumulate surfactant at the bottom of a bubble, which is con- trary to the macroscopic surface tension gradient ${ }^{17}$. The relative importance and influence of the two mechanisms is difficult to estimate, so no quantitative analysis is proposed.

Finally, it seems that in rising foam experiments the rigid formulations lead to drainage with fluid boundary conditions as at the bottom of the column the foam is wet and the DOH has not had time to adsorb onto the interfaces. This emphases that, in bubbling experiments, the permeability is set at the bottom of the foam. This is because the liquid is incorporated in the foam at the bottom so a faster drainage limits the whole water uptake.

\section{Conclusion}

We have studied the liquid fraction profile in rising foam. We use a classical model of foam drainage with Darcy's law. The pressure gradient includes both gravity and osmotic pressure, and through the permeability we account for the mobility of the interfaces using recent expressions obtained from forced drainage experiments. The model describes experimental data on systems with mobile interfaces very well. We show that the liquid fraction depends very weakly on the ordering at the bottom of the foam and that in bidisperse bubble distributions the larger bubble sizes control the foam drainage.

The model predicts higher liquid fractions if the interfaces are rigid. However, in our experiments systems which behave as rigid in forced drainage, behave as mobile ones. This can be explained by considering that the relative rigidity of an interface is influenced both by the surface viscosity and the liquid fraction (via the Boussinesq number). Bubbling to make foam means new interfaces and high liquid fractions at the bottom of the column. The combination of unfinished adsorption of dodecanol and large Plateau border radii make for fast drainage. The fast drainage at the bottom of the foam fixes the liquid flux leading to dryer foam than could have been expected.

Through these results we recall once more that the concept of mobile or rigid interfaces should treated with caution as it is not only dependent on the physical chemistry of the interfaces, but also on the problem under consideration. Thus a system that behaves as rigid in one problem can behave as mobile in another.

\section{Acknowledgements}

We thank I. Cantat, M.-C. Jullien, D. Langevin and O. Pitois for fruitful discussion. We acknowledge a financial support from the French space agency CNES.

\section{A Experimental uncertainties of $\varepsilon$}

Electrical conductivity measurements provide us with an accurate estimation of the liquid fraction, the position of electrodes can also be measured from photographs with sufficiently high precision. The main source of errors in our experiments is the distribution of bubble sizes. Typically the bubble size distribution is monodisperse but it still has a certain standard deviation $\delta$. To estimate the influence of this slight polydispersity on our predictions we took the experimental data shown in Figure 3 and added theoretical liquid fraction profiles for three different bubble radii: $R_{32}$ and $R_{32} \pm \delta$ (see Figure 9).

One can see that the experimental data lie between two limits. 


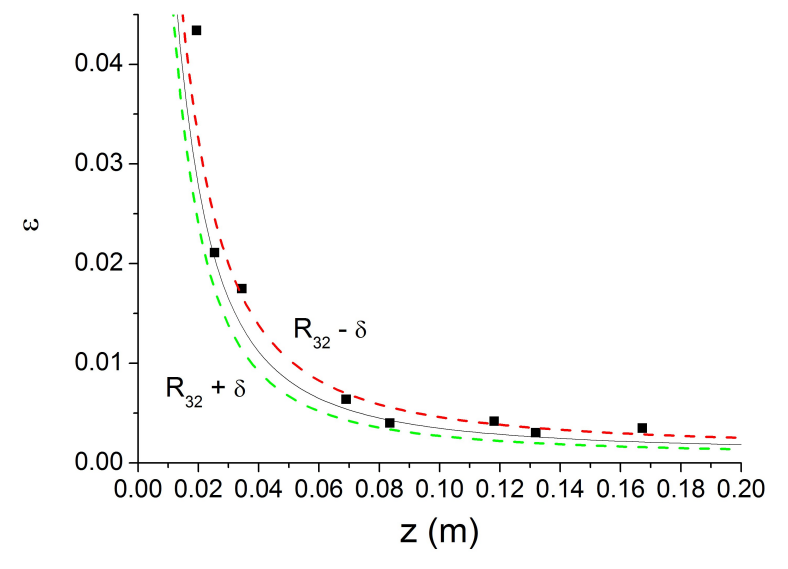

Fig. 9 Liquid fraction profile for SDS12 foam produced at $10 \mathrm{ml} / \mathrm{min}$ gas flow rate. Black points represent experimental data, the lines show calculated profiles for different bubble sizes: $R_{32}=523 \mu \mathrm{m}$ (black line), $R_{32}-\delta=473 \mu \mathrm{m}$ (red dashed line), $R_{32}+\delta=573 \mu \mathrm{m}$ (green dashed line)

So the dispersion of bubble sizes can indeed explain the scattering in the experimental points.

\section{B Gradient of the osmotic pressure}

We could also imagine that the change of surface tension influences the osmotic pressure gradient. To evaluate this effect, we take into account the surface tension gradient and write the osmotic pressure such as:

$$
\vec{\nabla} \Pi=\gamma \vec{\nabla}\left[\alpha \frac{\left(\varepsilon_{\max }-\varepsilon\right)^{2}}{\sqrt{\varepsilon} R}\right]+\left[\alpha \frac{\left(\varepsilon_{\max }-\varepsilon\right)^{2}}{\sqrt{\varepsilon} R}\right] \vec{\nabla} \gamma .
$$

The numerical estimations show that the second term can always be neglected in our experiments, and that we can use the equilibrium value of the surface tension to calculate the osmotic pressure.

\section{References}

1 Isabelle Cantat, Sylvie Cohen-Addad, Florence Elias, Francois Graner, Reinhard HÂühler, and Olivier Pitois. Foams: structure and dynamics. Oxford University Press, 2013.

2 Isabelle Cantat and Benjamin Dollet. Liquid films with high surface modulus moving in tubes: dynamic wetting film and jumpy motion. Soft Matter, 8(30):7790-7796, 2012.

3 Sylvie Cohen-Addad, Reinhard Höhler, and Olivier Pitois. Flow in foams and flowing foams. Annual Review of Fluid Mechanics, 45(1):241, 2013.

4 W Drenckhan, H Ritacco, Arnaud Saint-Jalmes, A Saugey, P McGuinness, A Van der Net, Dominique Langevin, and Denis Weaire. Fluid dynamics of rivulet flow between plates. Physics of Fluids (1994-present), 19(10):102101, 2007.

5 Wiebke Drenckhan and Arnaud Saint-Jalmes. The science of foaming. Advances in Colloid and Interface Science, (0), 2015.

6 K. Feitosa and D.J. Durian. Gas and liquid transport in steady-state aqueous foam. The European Physical Journal E, 26(3):309-316, 2008.

7 K Feitosa, S Marze, A Saint-Jalmes, and DJ Durian. Electrical conductivity of dispersions: from dry foams to dilute suspensions. Journal of Physics: Condensed Matter, 17(41):63016305, OCT 192005.

8 Alfonso M. Ganan-Calvo. Perfectly monodisperse microbubbling by capillary flow focusing: An alternate physical description and universal scaling. Phys. Rev. E, 69:027301, Feb 2004.

9 R. Hohler, Y. Yip Cheung Sang, E. Lorenceau, and S. CohenAddad. Osmotic pressure and structures of monodisperse ordered foam. Langmuir, 24(2):418-425, 2008.

10 Shu-Hao Hsu, Wei-Hua Lee, * Yu-Min Yang, Chien-Hsiang Chang, , and Jer-Ru Maa. Bubble formation at an orifice in surfactant solutions under constant-flow conditions. Industrial \& Engineering Chemistry Research, 39(5):1473-1479, 2000.

11 S. Hutzler, S. T. Tobin, A. J. Meagher, A. Marguerite, and D. Weaire. A model system for foam fractionation. Proceedings of the Royal Society A-mathematical Physical and Engineering Sciences, 469(2154):20120727, June 2013.

12 Stefan Hutzler, Dörte Lösch, Enda Carey, Denis Weaire, Matthias Hloucha, and Cosima Stubenrauch. Evaluation of a steady-state test of foam stability. Philosophical Magazine, 91(4):537-552, 2011.

13 S.A. Koehler, S Hilgenfeldt, and H.A. Stone. Liquid flow through aqueous foams: The node-dominated foam drainage equation. Phys. Rev. Lett., 82(21):4232-4235, MAY 241999.

14 S.A. Koehler, S. Hilgenfeldt, and H.A. Stone. A generalized view of foam drainage: Experiment and theory. Langmuir, 16(15):6327-6341, 2000.

15 AA Kulkarni and JB Joshi. Bubble formation and bubble rise velocity in gas-liquid systems: A review. INDUSTRIAL \& ENGINEERING CHEMISTRY RESEARCH, 44(16):5873-5931, AUG 3 2005.

16 Ralph A Leonard and Robert Lemlich. A study of interstitial liquid flow in foam. part i. theoretical model and application 
to foam fractionation. AIChE journal, 11(1):18-25, 1965.

17 V.G. Levich. Physicochemical hydrodynamics. Prentice-Hall, 1962.

18 E Lorenceau, N Louvet, F Rouyer, and O Pitois. Permeability of aqueous foams. The European Physical Journal E, 28(3):293304, 2009.

19 Armando Maestro, Wiebke Drenckhan, Emmanuelle Rio, and Reinhard Hohler. Liquid dispersions under gravity: volume fraction profile and osmotic pressure. Soft Matter, 9:25312540, 2013.

20 Z. Mitrinova, S. Tcholakova, Z. Popova, N. Denkov, Bivash R. Dasgupta, and K. P. Ananthapadmanabhan. Efficient control of the rheological and surface properties of surfactant solutions containing c8âĂŞc18 fatty acids as cosurfactants. Langmuir, 29(26):8255-8265, 2013.

21 S J Neethling, H T Lee, and J J Cilliers. The recovery of liquid from flowing foams. Journal of Physics: Condensed Matter, 15(10):1563, 2003.

22 S.J. Neethling, H.T. Lee, and J.J. Cilliers. A foam drainage equation generalized for all liquid contents. Journal of Physics: Condensed Matter, 14(3):331-342, JAN 282002.

23 F. Rouyer, O. Pitois, E. Lorenceau, and N. Louvet. Permeability of a bubble assembly: From the very dry to the wet limit. Physics of Fluids, 22(4), APR 2010.

24 A. Saint-Jalmes, M. U. Vera, and D. J. Durian. Uniform foam production by turbulent mixing: new results on free drainage vs. liquid content. The European Physical Journal B - Condensed Matter and Complex Systems, 12(1):67-73, 1999.

25 A. Saint-Jalmes, M. U. Vera, and D. J. Durian. Uniform foam production by turbulent mixing: new results on free drainage vs. liquid content. The European Physical Journal B - Condensed Matter and Complex Systems, 12(1):67-73, 1999.

26 Arnaud Saint-Jalmes. Physical chemistry in foam drainage and coarsening. Soft Matter, 2(10):836-849, 2006.

27 P. Stevenson. The wetness of a rising foam. Industrial and Engineering Chemistry Research, 45(2):803-807, 2006.

28 P. Stevenson. Hydrodynamic theory of rising foam. Minerals Engineering, 20(3):282 - 289, 2007.

29 P. Stevenson and C. Stevanov. Effect of rheology and interfacial rigidity on liquid recovery from rising froth. Industrial \& Engineering Chemistry Research, 43(19):6187-6194, 2004.

30 P. Stevenson, C. Stevanov, and G.J. Jameson. Liquid overflow from a column of rising aqueous froth. Minerals Engineering, 16(11):1045 - 1053, 2003.

31 Paul Stevenson, editor. Foam Engineering: Fundamentals and Applications. Wiley, 2012.

32 G Verbist, D Weaire, and AM Kraynik. The foam drainage equation. Journal of Physics: Condensed Matter, 8(21):37153731, MAY 201996. 